\title{
Meta-analysis of longitudinal epigenome-wide association studies of military cohorts reveals multiple CpG sites associated with post-traumatic stress disorder
}

Clara Snijders 1,", Adam X. Maihofer ${ }^{2,3,4, *}$, Andrew Ratanatharathorn ${ }^{5}$, Dewleen G. Baker ${ }^{2,3,6}$, Marco P. Boks $^{7}$, Elbert Geuze ${ }^{7,8}$, Sonia Jain ${ }^{9}$, Ronald C. Kessler ${ }^{10}$, Ehsan Pishva ${ }^{1,11}$, Victoria B. Risbrough ${ }^{2,3,4}$, Murray B. Stein ${ }^{2,6,12}$, Robert J. Ursano ${ }^{13}$, Eric Vermetten ${ }^{14,15,16,17}$, Christiaan H. Vinkers ${ }^{18,19}$, PGC PTDS EWAS Consortium, Alicia K. Smith ${ }^{20,21}$, Monica Uddin ${ }^{22}$, Bart P. F. Rutten ${ }^{1, \uparrow}$, Caroline M. Nievergelt $t^{2,3,4, \dagger}$

\section{AFFILIATIONS}

${ }^{1}$ Maastricht University, School for Mental health and Neuroscience, Department of Psychiatry and Neuropsychology, Maastricht, Limburg, NL. ${ }^{2}$ University of California San Diego, Department of Psychiatry, La Jolla, CA, US. ${ }^{3}$ Veterans Affairs San Diego Healthcare System, Center of Excellence for Stress and Mental Health, San Diego, CA, US. ${ }^{4}$ Veterans Affairs San Diego Healthcare System, Research Service, San Diego, CA, US. ${ }^{5}$ Harvard T.H. Chan School of Public Health, Department of Epidemiology, Boston, MA, US. ${ }^{6}$ Veterans Affairs San Diego Healthcare System, Psychiatry Service, San Diego, CA, US. ${ }^{7}$ UMC Utrecht Brain Center, Department of Psychiatry, Utrecht, Utrecht, NL. ${ }^{8}$ Netherlands Ministry of Defense, Brain Research \& Innovation Centre, Utrecht, Utrecht, NL.

${ }^{9}$ University of California San Diego, Department of Family Medicine and Public Health, La Jolla, CA, US. ${ }^{10}$ Harvard Medical School, Department of Health Care Policy, Boston, MA, US. ${ }^{11}$ University of Exeter Medical School, College of Medicine and Health, Exeter, UK. ${ }^{12}$ Veterans Affairs San Diego Healthcare System, Million Veteran Program, San Diego, CA, US. ${ }^{13}$ Uniformed Services University, Department of Psychiatry, Bethesda, Maryland, US. ${ }^{14}$ Arq, Psychotrauma Research Expert Group, Diemen, NH, NL. ${ }^{15}$ Leiden University Medical Center, Department of Psychiatry, Leiden, ZH, NL. ${ }^{16}$ Netherlands Ministry of Defense, Military Mental Healthcare, Utrecht, UT, NL. ${ }^{17}$ New York University School of Medicine, Department of Psychiatry, New York, NY, US. ${ }^{18}$ Amsterdam UMC (location VUmc), Department of Anatomy and Neurosciences, Amsterdam, Holland, NL.

${ }^{19}$ Amsterdam UMC (location VUmc), Department of Psychiatry, Amsterdam, Holland, NL. ${ }^{20}$ Emory University, Department of Psychiatry and Behavioral Sciences, Atlanta, GA, US. ${ }^{21}$ Emory University, Department of Gynecology and Obstetrics, Atlanta, GA, US ${ }^{22}$ University of South Florida College of Public Health, Genomics Program, Tampa, FL, US

\footnotetext{
* These authors contributed equally to this work

$\uparrow$ These authors share senior authorship

Corresponding author: Caroline Nievergelt (cnievergelt@ucsd.edu)
} 


\section{Abstract}

Background: Epigenetic mechanisms have been suggested to play a role in the development of post-traumatic stress disorder (PTSD). Here, blood-derived DNA methylation data (HumanMethylation450 BeadChip) collected prior to and following combat exposure in three cohorts composed of male military members were combined to assess whether DNA methylation profiles are associated with the development of PTSD. Methods: A total of 123 cases and 143 trauma-exposed controls were included. The Psychiatric Genomics Consortium (PGC) PTSD EWAS QC pipeline was used on all cohorts, and results were combined using a sample size weighted meta-analysis. We first combined two cohorts in a discovery stage $(\mathrm{N}=126$ and 78), sought targeted replication in the third cohort $(\mathrm{N}=62)$ and then performed a meta-analysis of all three datasets. Results: The discovery stage identified four CpG sites in which, conditional on pre-deployment DNA methylation, post-deployment DNA methylation was associated with PTSD status after adjustment for multiple comparisons. The most significant $\mathrm{CpG}\left(p=1.0 \times 10^{-08}\right)$ was located on $5 \mathrm{q} 31$ and replicated in the third cohort. When combining all cohorts, this intergenic site remained most significant along with two CpGs located in MADIL1 and HEXDC. Interestingly, the CpG site of MAD1L1 had an underlying single nucleotide polymorphism (SNP) which was located within the same LD block as a recently identified PTSD-associated SNP. Twelve differential methylated regions (DMRs) were also identified, one of which was located in MAD1L1 and four were situated in the human leukocyte antigen (HLA) region. Conclusion: This study suggests that the development of PTSD is associated with distinct methylation patterns in several genomic positions and regions. Our most prominent finding points to the involvement of MADIL1 which was previously associated with PTSD.

Keywords: EWAS, longitudinal, DNA methylation, meta-analysis, trauma, PTSD, epigenetics 


\section{Introduction}

Post-traumatic stress disorder (PTSD) is a debilitating psychiatric disorder that can develop following direct or indirect exposure to a potentially life-threatening traumatic incident. Symptoms include persistent re-experiencing of the trauma, avoidance behavior, hyperarousal and negative mood [1]. Although most individuals have the potential to withstand negative effects of trauma exposure on long-term mental health and to recover promptly, some are more vulnerable and at increased risk of developing PTSD. Understanding the molecular and neurobiological underpinnings of this differential susceptibility is currently receiving considerable attention, and epigenetic mediation of environmental influences has been proposed as a potential key mechanism [2-4].

Several epigenome-wide association studies (EWAS) have aimed to identify differentially methylated CpGs in PTSD [5-8]. However, most of these studies are based on association analyses where methylation was assessed at a single timepoint (cross-sectional), with limited ability to adjust for confounding variables. Only one PTSD study to date reported longitudinal changes in methylation profiles across a period of combat exposure in order to capture changes in DNA methylation over time in relation to phenotypic changes [7].

Here, we aimed to extend these analyses with two additional independent, yet highly similar military cohorts $[9,10]$. DNA and phenotypic data for all 3 male cohorts were collected prior to and following a 4-7 months deployment to an active ware zone in Iraq or Afghanistan. All studies selected PTSD cases and controls at post-deployment and only included subjects without PTSD at pre-deployment. We followed a two-stage design where we first combined two of these studies in order to identify longitudinal associations between DNA methylation and PTSD development. Data from the third, previously analyzed cohort [7], was used to replicate the obtained findings in a targeted manner. The second stage consisted of performing a meta-analysis across all three studies. Of the significant CpGs, we 
assessed associations with nearby single nucleotide polymorphisms (SNPs) and gene expression data, and examined correlations between blood and brain methylation status. To the best of our knowledge, this is the largest meta-analysis aimed at detecting methylation changes associated with the development of PTSD. This approach permits us to more accurately capture dynamic changes in DNA methylation in relation to PTSD development while minimizing confounding due to intra-individual variability.

\section{Methods and materials}

\section{Discovery datasets}

\section{Marine Resiliency Study}

The Marine Resiliency Study (MRS) [9] is a prospective PTSD study of Marines and Navy personnel deployed to Iraq or Afghanistan. PTSD symptoms were assessed approximately one month before deployment, three and/or six months post-deployment using the ClinicianAdministered PTSD scale (CAPS) and the PTSD Checklist (PCL) for DSM-IV. Biological samples including whole blood were collected at all time points. Information on smoking and alcohol use was collected on a self-report basis. Combat exposure was assessed approximately one week post-deployment using the Deployment Risk and Resilience Inventory (DRRI). A subset of 63 PTSD cases and 63 controls was selected for the methylation assays and inclusion in the present study. All subjects were free of a PTSD diagnosis at pre-deployment and had CAPS scores $\leq 25$. After return from a $\sim 7$-months deployment period, PTSD cases (following the DSM-IV full or partially stringent diagnosis $[11,12]$ ) were selected either at the three- or the six-month follow-up visit, based on when these subjects had their highest recorded CAPS scores. Subsequently, controls were frequency matched to the selected cases for age, ancestry, and time of post-deployment visit. The study was approved by the 
institutional review boards of the University of California San Diego, VA San Diego

Research Service, and Naval Health Research Center. All subjects provided informed consent.

\section{Army STARRS}

The Army Study to Assess Risk and Resilience in Servicemembers (Army STARRS) is a prospective study among U.S. Army personnel gathering information on risk and resilience factors for suicidality and psychopathology [10]. All subjects completed the PCL6 screener for DSM-IV approximately 6 weeks before deployment to Afghanistan and the PCL-C at one, two, and six months post-deployment. PTSD diagnosis was assigned using multiple imputation methods [13] and information on trauma exposure was gathered from selfadministered questions on childhood, adult, and military-related events. Information on smoking and alcohol use was collected on a self-report basis. Biological samples including whole blood were collected approximately 6 weeks before deployment and one month postdeployment. A subset of 31 cases and 47 controls were selected for the methylation assays and inclusion in this analysis. All subjects were free of a PTSD diagnosis at pre-deployment. PTSD cases were selected based on their PTSD diagnosis at 6 months post-deployment. Controls were PTSD-free subjects matched on age, deployment stress and childhood adversity. The study procedures were approved by the Institutional Review Boards of all collaborating organizations. All subjects provided informed consent.

\section{Replication dataset: PRISMO}

Replication data was obtained from the Prospective Research In Stress-related Military Operations (PRISMO) study, a prospective study of Dutch military soldiers deployed to Afghanistan $[14,15]$. The severity of current PTSD symptoms was assessed using the SelfReport Inventory for PTSD (SRIP) and blood samples were collected approximately one 
month before and one and six months after deployment. Traumatic stress exposure during deployment to Afghanistan was assessed with a deployment experiences checklist. Information on smoking and alcohol use was collected on a self-report basis. A subset of 29 cases and 33 controls was selected for the methylation assays and inclusion in this analysis. The study was approved by the ethical committee of the University Medical Center Utrecht, and was conducted in accordance with the Declaration of Helsinki. All subjects provided informed consent.

\section{Quality control}

In all cohorts, longitudinal whole blood DNA methylation levels were measured using the Illumina HumanMethylation450K BeadChip. The Psychiatric Genomics Consortium (PGC)EWAS quality control pipeline was used on all three cohorts [5]. Briefly, samples were excluded when having a probe detection call rate $<90 \%$ and an average intensity value $<50 \%$ of the overall sample mean or $<2,000$ arbitrary units (AU). Individual probes with detection $p$ values $>0.001$ or those based on less than three beads were set to missing. Remaining probes were excluded when cross-reactivity occurred between autosomal and sex chromosomes. CpG sites with missing data for $>10 \%$ of samples within cohorts were excluded. After filtering, the $\beta$-values reflecting methylation levels of individual cytosine residues were normalized to correct for differences between type I and type II probes using Beta Mixture Quantile Normalization (BMIQ) [16]. ComBat [17] was used to correct for remaining issues such as batch and plate effects. To account for differences in cell type composition between samples, proportions of $\mathrm{CD} 8, \mathrm{CD} 4, \mathrm{NK}, \mathrm{B}$ cells, monocytes and granulocytes were estimated for each individual using their unique DNA methylation profiles.

\section{Statistical analysis}


The normalized $\beta$-values were logit transformed to M-values which were used for linear regression analysis. Post-deployment DNA methylation was modeled as a function of postdeployment PTSD status while adjusting for pre-deployment DNA methylation, age, changes in CD4T, CD8T, NK, B cell, and monocyte cell proportions, and principal components (PCs) for ancestry. For MRS and Army STARRS, the PCs were derived from available genome wide association studies (GWAS) and PCs 1-3 were included. For PRISMO, the method described by Barfield and colleagues [18] was used to derive PCs from the EWAS data and PCs 2-4 (see [5]) were included. HC3 standard errors were calculated using the sandwich R library [19]. Analyses were performed on each cohort independently and the obtained $p$ values were combined using a sample size weighted meta-analysis. Significance was declared at $p<1.03 \times 10^{-7}$ after a stringent Bonferroni correction for 439,897 probes. Possible confounding effects of changes in smoking and alcohol use were assessed as a sensitivity analysis.

Differential methylated regions (DMR) analysis was performed on a set of 26,000 predefined gene regions within gene bodies, promoter regions, and $\mathrm{CpG}$ islands using the mCSEA version 1.2 package for $\mathrm{R}$ [20]. Regions were included when annotated to having at least 5 CpGs. For each study, EWAS p-values, methylation level values, and a phenotype and covariate data matrix were supplied as program inputs. P-values were derived using 100,000 permutations. A sample size weighted meta-analysis of DMRs was performed based on zscore transformations of permutation $p$-values. Significances of DMRs $\left(p<1.92 \times 10^{-6}\right)$ were derived based on a Bonferroni correction for the 26,000 tests performed. All positions and regions were in reference to GRCh37/hg19.

Detecting genetic effects and links with gene expression 
Associations between baseline levels of methylation of each significant $\mathrm{CpG}$ from the second analysis stage and nearby SNPs (within 500 kilobases; kb) were assessed in the MRS dataset using PLINK [21] to detect the potential influence of genetic effects on DNA methylation. For a given $\mathrm{CpG}$ site, the SNP with the lowest $p$-value was carried forward as an additional covariate in the regression models as a sensitivity analysis.

For CpGs annotated to genes, we estimated the correlation between $\mathrm{CpG}$ methylation levels and blood gene expression in MRS data. Details of messenger RNA (mRNA) expression measurement in MRS can be found elsewhere [22].

We used the UCSC genome browser tool (http://genome.ucsc.edu/) to identify if SNPs associated with our CpGs influenced expression in other tissue types based on combined expression eQTL data from 44 tissues from GTEx v6 [23].

\section{Blood-brain correlations}

The Blood Brain DNA Methylation Comparison Tool (http://epigenetics.iop.kcl.ac. uk/bloodbrain/) was used to assess correlations between the methylation status of the top hits of the combined meta-analysis in blood and brain [24]. Specifically, this tool yields Pearson's correlation coefficients $(r)$ and associated $p$-values for the association of the methylation status of individual $\mathrm{CpG}$ sites in blood and the prefrontal cortex, entorhinal cortex, superior temporal gyrus, and cerebellum.

\section{Results}

\section{Cohorts}

Demographic and clinical characteristics of subjects from all three cohorts (total $\mathrm{N}$ subjects $=$ 266) can be found in Table 1. All subjects were male and the majority were of European ancestry $(\mathrm{N}=211,79 \%)$. Within each cohort, cases and controls did not differ significantly in 
terms of age. Pre-deployment PTSD symptoms were significantly different between cases and controls from MRS only, with cases scoring slightly higher on the CAPS as compared to controls ( $p=.002$; Table 1). In MRS and Army STARRS, cases were exposed to more traumatic events as compared to controls ( $p<.001$ for both cohorts).

\section{Discovery stage: meta-analysis of MRS and Army STARRS}

A meta-analysis of MRS and Army STARRS was performed to identify CpG sites with methylation changes associated with PTSD at post-deployment. Four genome-wide significant CpG sites (i.e. differentially methylated positions, DMPs) were identified using a Bonferroni threshold of $p=1.03 \times 10^{-07}$ (Table 2). These sites were located near SPRY4, in SDK1, CTRC and CDH15, respectively. The direction of DNA methylation profiles associated with PTSD development was different for each site (Supplemental Figures S1-4). After Bonferroni correction for $~ 26,000$ predefined regions, 19 DMRs were identified in which longitudinal changes in DNA methylation were associated with PTSD (Table 3).

\section{Replication in PRISMO}

The association of one CpG site, the intergenic site cg05656210, was nominally replicated in PRISMO ( $p=2.0 \times 10^{-02}$; Table 2 ), with both the discovery meta-analysis and replication analysis showing decreased DNA methylation in association with PTSD development. None of the 19 significant DMRs were replicated in PRISMO (Table 3).

\section{Meta-analysis across all cohorts}

When combining MRS, Army STARRS and PRISMO, the DNA methylation profile of three CpG sites was significantly associated with post-deployment PTSD status (Table 2, Figure 1). The intergenic $\mathrm{CpG}$ that replicated in PRISMO remained the most significant $(\mathrm{Z}=-6.14, p=$ 
$\left.8.1 \times 10^{-10}\right)$. The other sites were located in the gene body regions of MADIL1 and HEXDC (Supplemental Figures S1, S5, S6). Sensitivity analyses for the potentially confounding effects of changes in smoking and alcohol use did not substantially affect these results (data not shown). Furthermore, 12 DMRs were associated with PTSD (Supplemental Figures S718, Figure 1). Seven of these were also significant in the discovery stage, and four were located in the human leukocyte antigen (HLA) region (Table 3).

Genetic effects and gene expression

Using MRS data, genetic effects on DNA methylation levels of the significant DMPs were assessed by testing for associations with SNPs within $500 \mathrm{~kb}$ of the DMPs. All DMPs had significantly associated SNPs which explained approximately $80 \%$ of the variation in methylation $\left(p<2 \times 10^{-16}\right)$ and were located within $1 \mathrm{bp}$ of their respective $\mathrm{CpG}$ sites (Supplemental Table S1). However, adjusting for genotypes in the main model to assess the impact of SNPs on changes in DNA methylation over time did not significantly affect the observed findings (Supplemental Table S2). We further assessed the association between methylation signatures of these DMPs and blood-derived gene expression data which was available for MRS [25]. To do so, DNA methylation levels were averaged across time points. Methylation levels of the CpGs located in HEXDC and MADIL1 were significantly correlated with gene expression data (Table 4), with an inverse correlation between DNA methylation and expression in HEXDC and a positive correlation between methylation and expression of MAD1L1. Methylation of intergenic site cg05656210 was not significantly associated with expression of the most nearby gene, SPRY4.

Blood-brain correlations of PTSD-associated CpGs 
Blood-brain correlations of methylation levels of the significant stage 2 DMPs were examined using a publicly available database [24]. For all three DMPs, blood DNA methylation levels correlated strongly with those in the prefrontal cortex, entorhinal cortex, superior temporal gyrus, and cerebellum ( $r \geq 0.93$ for all DMPs; $p$-values ranging between $1.48 \times 10^{-32}$ and 5.32 x 10-72; Supplemental Table S3, Supplemental Figure 3 for cg05656210).

\section{Discussion}

Exposure to trauma is a prerequisite for the development of PTSD, yet not all individuals develop PTSD following trauma [26]. The underlying biological mechanisms of this differential susceptibility have not yet been fully identified and even the largest genome-wide association studies to date explain only a small proportion of the disease liability $[27,28]$. Epigenetic changes have been studied as one potential mechanism, but most association studies have used cross-sectional designs which render it impossible to establish causality. Here, we are using a more powerful longitudinal design to investigate changes in DNA methylation from pre- to post-combat exposure across very similar military cohorts deployed to combat in Iraq and Afghanistan. We started with a meta-analysis across the US-based MRS and Army STARRS cohorts and sought replication using the previously published Dutch PRISMO study [7]. To increase power, we also performed a meta-analysis across all 3 cohorts. The discovery stage meta-analysis of two studies revealed four genome-wide significant DMPs and 19 DMRs which were linked to PTSD development. One of these DMPs replicated in PRISMO. In a combined meta-analysis of all three studies, the replicating DMP and 7 DMRs remained significant, and 2 additional DMPs and 12 DMRs were significantly associated with PTSD development.

Follow-up analyses were done using the significant DMPs from the combined metaanalysis. The replicating DMP cg05656210 remained the top-ranked significant marker in the 
second stage. cg05656210 is an intergenic site annotated near SPRY4. SPRY4 was previously found differentially methylated in blood of patients diagnosed with schizophrenia [29] and has further been shown to interact with $S K A 2$ [30], a gene suggested to be a promising biomarker for suicidal behavior [31, 32], stress susceptibility and stress-related disorders such as PTSD [32-34].

The second top significant probe, $\operatorname{cg} 12169700$, is located in MADIL1, a gene involved in cell cycle control that has previously been associated in a GWAS of bipolar disorder [35, 36], schizophrenia [36, 37] and depression [38]. One of the significant DMRs was also located within this gene. Interestingly, MADILI was recently identified in a PTSD GWAS of the Million Veteran Program (MVP) [27]. cg12169700 is a CpG site that overlaps with a common SNP (CpG-SNP). This underlying SNP, rs11761270, is located in the same large linkage disequilibrium (LD) block as the MVP MAD1L1 finding. In the MVP, carriers of the minor allele of rs11761270 showed decreased levels of methylation and were at increased risk of having PTSD. This corresponds to our own findings in which PTSD cases show a reduction in methylation from pre- to post-deployment. Moreover, using expression data from MRS, we found that methylation at this site was positively associated with gene expression of MADILI. This also aligns with previous findings that showed that blood levels of MAD1L1 were decreased in highly stress-susceptible individuals [39]. Together, these findings suggest that specific methylation profiles within MADIL1 may be regarded as a risk factor for PTSD in addition to several other psychiatric disorders [40].

The third CpG site is located in HEXDC which to date has no known implications in any psychiatric disease. The DMP of HEXDC was located directly adjacent to rs4789774, a known expression quantitative trait locus (eQTL) that regulates the expression of HEXDC in the human brain cortex and of NARF and NARF-ITI in a number of tissue types including 
blood (http://genome.ucsc.edu/). Moreover, a modest negative correlation was found between methylation of this site and gene expression of $H E X D C$.

The discovery that methylation levels at the top three PTSD-associated CpGs were highly associated with the genotype of the nearby SNPs led us to question whether the associations between methylation and PTSD status were mainly driven by genotype. However, direct adjustment for genotype in a sensitivity analysis did not attenuate the associations between DNA methylation and PTSD status. Our current sample size limits our ability to conduct analyses specific to genotype strata to further investigate interaction effects between SNPs and methylation.

Twelve significant DMRs were found in the second phase of the analysis. Our strongest finding was in the HLA region which encodes the major histocompatibility complex (MHC) and has repeatedly been implicated in neuropsychiatric disorders (recently reviewed in [41]).

Since our methylation data were based on DNA from peripheral blood, we further examined correlations between blood and several brain regions, i.e. the prefrontal cortex, the entorhinal cortex, superior temporal gyrus and cerebellum. The results indicate that bloodbrain correlations of all top CpGs were strong for all four brain regions suggesting that these findings could potentially also be relevant for tissues other than blood. Assessing these correlations is relevant when dealing with disorders such as PTSD which are characterized by functional and structural alterations within the brain but for which the accessibility to human brain tissue is limited. However, these and similar findings will need to be confirmed using postmortem brain tissue and their precise role in PTSD development will need to be investigated further.

The main limitation of the present study is its small sample size which likely captures only a fraction of all implicated CpGs and renders additional analyses such as pathway and network analyses underpowered. It further needs to be emphasized that this study used data 
generated with Illumina's 450K arrays which only assess a subset of all CpG sites. Next, although examining blood-derived DNA methylation is informative when seeking relatively easily accessible biomarkers, follow-up studies are needed in order to assess these methylation patterns within the tissue of interest, i.e. the brain. Furthermore, at this stage it is unclear whether the identified differential methylation patterns in PTSD cases have any functional consequences. Although they may influence gene expression, the current dataset has limited power to establish causality. Finally, to maximize power for discovery, the present cohorts were chosen to be highly similar in regards to demographics, type of trauma, and time since trauma exposure. Thus, the degree to which these findings on active duty, predominantly European-ancestry military men, may generalize to females, civilians, or other ancestries, is unclear.

In summary, this largest study on methylation changes associated with the development of PTSD to date points towards biologically interesting genes such as the HLA region and MADIL1, a PTSD-related gene recently identified in the large MVP, strengthening the notion that DNA methylation is involved in the development of PTSD. Larger longitudinal studies and integrative efforts are now needed to build upon these preliminary findings in order to understand their functional consequences and integrate them more broadly into our current understanding of the (epi)genomic basis of PTSD. 


\section{References}

1. Association, A.P., Diagnostic and statistical manual of mental disorders (5th ed.). 2013, Arlington, VA: American Psychiatric Publishing.

2. $\quad$ Nievergelt, C.M., et al., Genomic Approaches to Posttraumatic Stress Disorder: The Psychiatric Genomic Consortium Initiative. Biol Psychiatry, 2018. 83(10): p. 831-839.

3. Daskalakis, N.P., et al., Recent Genetics and Epigenetics Approaches to PTSD. Curr Psychiatry Rep, 2018. 20(5): p. 30.

4. Sheerin, C.M., et al., The Genetics and Epigenetics of PTSD: Overview, Recent Advances, and Future Directions. Curr Opin Psychol, 2017. 14: p. 5-11.

5. Ratanatharathorn, A., et al., Epigenome-wide association of PTSD from heterogeneous cohorts with a common multi-site analysis pipeline. Am J Med Genet B Neuropsychiatr Genet, 2017. 174(6): p. 619-630.

6. $\quad$ Kuan, P.F., et al., An epigenome-wide DNA methylation study of PTSD and depression in World Trade Center responders. Transl Psychiatry, 2017. 7(6): p. e1158.

7. $\quad$ Rutten, B.P.F., et al., Longitudinal analyses of the DNA methylome in deployed military servicemen identify susceptibility loci for post-traumatic stress disorder. Mol Psychiatry, 2018. 23(5): p. 1145-1156.

8. Smith, A.K., et al., Differential immune system DNA methylation and cytokine regulation in post-traumatic stress disorder. Am J Med Genet B Neuropsychiatr Genet, 2011. 156B(6): p. 700-8.

9. $\quad$ Baker, D.G., et al., Predictors of risk and resilience for posttraumatic stress disorder among ground combat Marines: methods of the Marine Resiliency Study. Prev Chronic Dis, 2012. 9: p. E97.

10. Ursano, R.J., et al., The Army study to assess risk and resilience in servicemembers (Army STARRS). Psychiatry, 2014. 77(2): p. 107-19.

11. Sher, L., Recognizing post-traumatic stress disorder. QJM: An International Journal of Medicine, 2004. 97(1): p. 1-5.

12. Smith, A.K., et al., Epigenome-wide meta-analysis of PTSD across 10 military and civilian cohorts identifies novel methylation loci. bioRxiv, 2019: p. 585109.

13. Kessler, R.C., et al., Clinical reappraisal of the Composite International Diagnostic Interview Screening Scales (CIDI-SC) in the Army Study to Assess Risk and Resilience in Servicemembers (Army STARRS). Int J Methods Psychiatr Res, 2013. 22(4): p. 303-21.

14. Reijnen, A., et al., Prevalence of mental health symptoms in Dutch military personnel returning from deployment to Afghanistan: a 2-year longitudinal analysis. Eur Psychiatry, 2015. 30(2): p. 341-6.

15. Eekhout, I., et al., Post-traumatic stress symptoms 5 years after military deployment to Afghanistan: an observational cohort study. Lancet Psychiatry, 2016. 3(1): p. 58-64.

16. Teschendorff, A.E., et al., A beta-mixture quantile normalization method for correcting probe design bias in Illumina Infinium 450 k DNA methylation data. Bioinformatics, 2013. 29(2): p. $189-96$.

17. Johnson, W.E., C. Li, and A. Rabinovic, Adjusting batch effects in microarray expression data using empirical Bayes methods. Biostatistics, 2007. 8(1): p. 118-27.

18. Barfield, R.T., et al., Accounting for population stratification in DNA methylation studies. Genet Epidemiol, 2014. 38(3): p. 231-41.

19. Zeileis, A., Econometric Computing with HC and HAC Covariance Matrix Estimators. Journal of Statistical Software, 2004. 11(10).

20. Martorell-Marugan, J., V. Gonzalez-Rumayor, and P. Carmona-Saez, mCSEA: Detecting subtle differentially methylated regions. 2018: p. 293381.

21. Purcell, S., et al., PLINK: a tool set for whole-genome association and population-based linkage analyses. American journal of human genetics, 2007. 81(3): p. 559-575.

22. Breen, M.S., et al., Gene networks specific for innate immunity define post-traumatic stress disorder. Molecular psychiatry, 2015. 20(12): p. 1538-1545.

23. Consortium, G.T., The Genotype-Tissue Expression (GTEx) project. Nature genetics, 2013. 45(6): p. 580-585. 
24. Hannon, E., et al., Interindividual methylomic variation across blood, cortex, and cerebellum: implications for epigenetic studies of neurological and neuropsychiatric phenotypes.

Epigenetics, 2015. 10(11): p. 1024-32.

25. Nievergelt, C.M., et al., Genomic predictors of combat stress vulnerability and resilience in U.S. Marines: A genome-wide association study across multiple ancestries implicates PRTFDC1 as a potential PTSD gene. (1873-3360 (Electronic)).

26. Yehuda, R., et al., Post-traumatic stress disorder. Nat Rev Dis Primers, 2015. 1: p. 15057.

27. Gelernter J., e.a., Genome-wide association study of posttraumatic stress disorder (PTSD) reexperiencing symptoms in >165,000 US veterans. Nature Neuroscience, 2019. In press.

28. Nievergelt, C.M., et al., Largest genome-wide association study for PTSD identifies genetic risk loci in European and African ancestries and implicates novel biological pathways. bioRxiv, 2018: p. 458562.

29. Zaharieva, I., et al., Association study in the 5q31-32 linkage region for schizophrenia using pooled DNA genotyping. BMC Psychiatry, 2008. 8: p. 11.

30. He, X.J., et al., Long non-coding RNA SPRY4-IT1 promotes the proliferation and invasion of U251 cells through upregulation of SKA2. Oncol Lett, 2018. 15(3): p. 3977-3984.

31. Guintivano, J., et al., Identification and replication of a combined epigenetic and genetic biomarker predicting suicide and suicidal behaviors. Am J Psychiatry, 2014. 171(12): p. 1287-96.

32. Kaminsky, Z., et al., Epigenetic and genetic variation at SKA2 predict suicidal behavior and post-traumatic stress disorder. Transl Psychiatry, 2015. 5: p. e627.

33. Boks, M.P., et al., SKA2 Methylation is Involved in Cortisol Stress Reactivity and Predicts the Development of Post-Traumatic Stress Disorder (PTSD) After Military Deployment. Neuropsychopharmacology, 2016. 41(5): p. 1350-6.

34. Sadeh, N., et al., SKA2 methylation is associated with decreased prefrontal cortical thickness and greater PTSD severity among trauma-exposed veterans. Mol Psychiatry, 2016. 21(3): p. 357-63.

35. Trost, S., et al., Investigating the Impact of a Genome-Wide Supported Bipolar Risk Variant of MADIL1 on the Human Reward System. Neuropsychopharmacology, 2016. 41(11): p. 267987.

36. Zhao, L., et al., Replicated associations of FADS1, MAD1L1, and a rare variant at 10q26.13 with bipolar disorder in Chinese population. Transl Psychiatry, 2018. 8(1): p. 270.

37. Ripke, S., et al., Genome-wide association analysis identifies 13 new risk loci for schizophrenia. Nat Genet, 2013. 45(10): p. 1150-9.

38. Wray, N.R., et al., Genome-wide association analyses identify 44 risk variants and refine the genetic architecture of major depression. Nat Genet, 2018. 50(5): p. 668-681.

39. Le-Niculescu, H., et al., Towards precision medicine for stress disorders: diagnostic biomarkers and targeted drugs. Molecular Psychiatry, 2019.

40. Levey, D.F., et al., Reproducible Risk Loci and Psychiatric Comorbidities in Anxiety: Results from 200,000 Million Veteran Program Participants. bioRxiv, 2019: p. 540245.

41. Debnath, M., et al., The MHC/HLA Gene Complex in Major Psychiatric Disorders: Emerging Roles and Implications. Current Behavioral Neuroscience Reports, 2018. 5(2): p. 179-188. 


\section{ACKNOWLEDGMENTS}

This work was supported by the U.S. Army Medical Research and Materiel Command as well as the National Institute of Mental Health (NIMH; R01MH108826; R01MH106595). Funding for MRS was provided by the Marine Corps, Navy Bureau of Medicine and Surgery (BUMED), VA Health Research and Development (HSR\&D), Veterans Affairs San Diego Healthcare System, Center of Excellence for Stress and Mental Health, and NIH R01MH093500. Acknowledged are Mark A. Geyer (UCSD), Daniel T. O’Connor (UCSD), all MRS investigators, as well as the MRS administrative core and data collection staff. The authors also thank the Marine and Navy Corpsmen volunteers for military service and participation in MRS.

Data collection of PRISMO was funded by the Dutch Ministry of Defense, and DNA methylation analyses were funded by the VENI Award fellowship from the Netherlands Organization for Scientific Research (NWO, grant number 916.11.086).

Army STARRS was sponsored by the Department of the Army and funded under cooperative agreement number U01MH087981 (2009-2015) with the National Institutes of Health, National Institute of Mental Health (NIH/NIMH).

The views expressed in this article are those of the authors and do not necessarily reflect the position or policy of the VA, NIH, or the United States government.

\section{AUTHOR CONTRIBUTIONS}

PGC-PTSD management group:

C.M.N.

\section{Writing group:}

A.X.M., A.R., C.S., M.B.S.

\section{Study PI or co-PI:}

D.G.B., E.G., R.C.K., C.M.N., V.B.R., A.K.S., M.B.S., M.U., R.J.U., E.V.

\section{Obtained funding for studies:}

M.P.B., E.G., R.C.K., C.M.N., B.P.F.R., R.J.U., E.V.

\section{Clinical:}

D.G.B., E.G., E.V.

\section{Contributed data:}

D.G.B., S.J., V.B.R., M.B.S.

\section{Statistical analysis:}


M.P.B., A.X.M., C.M.N., E.P., B.P.F.R., C.H.V.

\section{Bioinformatics:}

M.P.B., A.X.M., A.R., B.P.F.R.

\section{Genomics:}

M.P.B., A.X.M., B.P.F.R., C.H.V.

\section{CONFLICT OF INTEREST}

R.C.K. received support for his epidemiological studies from Sanofi Aventis; was a consultant for Johnson \& Johnson Wellness and Prevention, Sage Pharmaceuticals, Shire, Takeda; and served on an advisory board for the Johnson \& Johnson Services Inc. Lake Nona Life Project. Kessler is a co-owner of DataStat, Inc., a market research firm that carries out healthcare research. M.B.S. has in the past three years been a consultant for Actelion, Aptinyx, Bionomics, Dart Neuroscience, Healthcare Management Technologies, Janssen, Neurocrine Biosciences, Oxeia Biopharmaceuticals, Pfizer, and Resilience Therapeutics. 


\section{Tables and figures}

Table 1. Demographics and clinical characteristics of MRS, Army STARRS and PRISMO

\begin{tabular}{|l|l|l|l|l|}
\hline & Cases & Controls & P-value & Overall \\
\hline N & & & & \\
\hline MRS & 63 & 63 & - & 126 \\
\hline Army STARRS & 31 & 47 & - & 78 \\
\hline PRISMO & 29 & 33 & - & 62 \\
\hline Age, mean (SD) & & & & \\
\hline MRS & $22.15(2.3)$ & $22.36(3.7)$ & .71 & $22.26(3)$ \\
\hline Army STARRS & $23.5(4.0)$ & $24.6(4.8)$ & .26 & $24.2(4.4)$ \\
\hline PRISMO & $27.1(9.9)$ & $27.1(8.7)$ & 1.0 & $27.1(9.0)$ \\
\hline PTSD pre-deployment, mean (SD) & & & & \\
\hline MRS & $10.8(7.5)$ & $6.8(6.5)$ & .002 & $8.8(7)$ \\
\hline Army STARRS & $7.4(2.6)$ & $6.8(2.0)$ & .40 & $7.0(2.2)$ \\
\hline PRISMO & $28.2(4.0)$ & $26.4(4.0)$ & .10 & $27.2(3.9)$ \\
\hline PTSD post-deployment, mean (SD) & & & & \\
\hline MRS & $58.17(13.5)$ & $13.36(6.1)$ & $<.001$ & $35.76(9.8)$ \\
\hline Army STARRS & $52.7(7.8)$ & $25.8(8.6)$ & $<.001$ & $36.5(8.1)$ \\
\hline PRISMO & $46.1(8.7)$ & $27.4(5.1)$ & $<.001$ & $36.1(6.5)$ \\
\hline Combat exposure, mean (SD) & & & & \\
\hline MRS & $1.08(0.8)$ & $0.66(0.4)$ & $<.001$ & $0.87(0.6)$ \\
\hline Army STARRS & $9.4(1.3)$ & $7.9(2.0)$ & $<.001$ & $8.5(1.7)$ \\
\hline PRISMO & $8.5(3.0)$ & $7.2(2.3)$ & .07 & $7.8(2.5)$ \\
\hline Ancestry, N (\%) & & & & \\
\hline MRS & & & & \\
\hline - European & $34(53)$ & $37(59)$ & & $71(56)$ \\
\hline - African & $5(8)$ & $5(8)$ & & $10(8)$ \\
\hline - Other & 24 & 21 & & $45(36)$ \\
\hline Army STARRS & & & & \\
\hline - European & $31(100)$ & $47(100)$ & & $78(100)$ \\
\hline PRISMO & & & & \\
\hline - European & $29(100)$ & $33(100)$ & & $62(100)$ \\
\hline For MRS, Army STARRS & & & \\
\hline
\end{tabular}

For MRS, Army STARRS and PRISMO, pre-deployment PTSD symptoms were measured using the Clinician-Administered PTSD Scale (CAPS), PTSD Checklist - screener (PCL-6) and SelfReport Inventory for PTSD (SRIP), respectively. Post-deployment PTSD symptoms were measured using the CAPS, PCL-C: PTSD Checklist - civilian version (PCL-C) and SRIP, respectively. Trauma exposure during combat was assessed using the Deployment Risk and Resilience Inventory (DRRI), specific items of the PCL, and the deployment experiences checklist, respectively. SD: standard deviation. 


\begin{tabular}{|c|c|c|c|c|c|c|c|c|c|c|c|c|c|c|c|c|}
\hline \multirow[b]{2}{*}{ Probe } & \multirow[b]{2}{*}{ Chr: position } & \multirow[b]{2}{*}{ Gene } & \multirow[b]{2}{*}{ Region } & \multicolumn{3}{|l|}{ MRS } & \multicolumn{3}{|c|}{ Army STARRS } & \multicolumn{2}{|c|}{$\begin{array}{l}\text { Discovery stage } \\
\text { meta-analysis }\end{array}$} & \multicolumn{3}{|c|}{ Replication in PRISMO } & \multicolumn{2}{|c|}{$\begin{array}{l}\text { Meta-analysis of } \\
3 \text { cohorts }\end{array}$} \\
\hline & & & & $\beta$ & SE & $P$-value & $\beta$ & SE & $P$-value & $\mathrm{Z}$ & $P$-value & $\beta$ & SE & $P$-value & Z & $P$-value \\
\hline $\operatorname{cg} 05656210$ & 5: 141660565 & Intergenic & Intergenic & -0.37 & 0.15 & $1.6 \mathrm{E}-02$ & -0.58 & 0.09 & $6.1 \mathrm{E}-10$ & -5.73 & 1.0E-08 & -0.47 & 0.20 & $2.0 \mathrm{E}-02 *$ & -6.14 & 8.1E-10 \\
\hline $\operatorname{cg} 12169700$ & 7: 1923695 & MADILI & Body & -1.24 & 0.27 & 4.2E-06 & -0.19 & 0.20 & 3.3E-01 & -4.22 & $2.4 \mathrm{E}-05$ & -0.64 & 0.14 & $4.3 \mathrm{E}-06$ & -5.91 & 3.3E-09 \\
\hline cg20756026 & 17: 80394529 & $H E X D C$ & Body & -0.62 & 0.21 & $3.3 \mathrm{E}-03$ & -0.28 & 0.09 & $2.6 \mathrm{E}-03$ & -4.17 & $3.0 \mathrm{E}-05$ & -0.37 & 0.09 & $2.6 \mathrm{E}-05$ & -5.69 & 1.3E-08 \\
\hline $\operatorname{cg} 16956686$ & 7: 4304779 & $S D K 1$ & Body & -0.19 & 0.04 & $3.6 \mathrm{E}-07$ & -0.13 & 0.05 & 7.1E-03 & -5.67 & 1.5E-08 & -0.04 & 0.09 & $6.3 \mathrm{E}-01$ & 0.63 & 2.0E-07 \\
\hline cg18917957 & 1: 15764093 & CTRC & TSS1500 & -0.34 & 0.08 & $2.3 \mathrm{E}-05$ & -0.26 & 0.08 & $4.9 \mathrm{E}-04$ & -5.48 & 4.2E-08 & -0.06 & 0.13 & $6.4 \mathrm{E}-01$ & 0.64 & 5.0E-07 \\
\hline $\operatorname{cg} 05901543$ & 16: 89251975 & CDH15 & Body & -0.14 & 0.03 & $2.4 \mathrm{E}-08$ & -0.06 & 0.03 & 7.1E-02 & -5.50 & 3.7E-08 & 0.01 & 0.05 & $8.2 \mathrm{E}-01$ & 0.82 & $2.5 \mathrm{E}-06$ \\
\hline
\end{tabular}




\begin{tabular}{|c|c|c|c|c|c|c|c|c|c|c|c|c|c|}
\hline \multirow[t]{2}{*}{ Chr: start-stop } & \multirow[t]{2}{*}{$\begin{array}{l}\# \text { of } \\
\text { probes }\end{array}$} & \multirow[t]{2}{*}{ Gene } & \multirow[t]{2}{*}{ Region } & \multicolumn{2}{|l|}{ MRS } & \multicolumn{2}{|c|}{ Army STARRS } & \multicolumn{2}{|c|}{$\begin{array}{l}\text { Discovery stage } \\
\text { meta-analysis }\end{array}$} & \multicolumn{2}{|c|}{$\begin{array}{l}\text { Replication in } \\
\text { PRISMO }\end{array}$} & \multicolumn{2}{|c|}{$\begin{array}{l}\text { Meta-analysis of } 3 \\
\text { cohorts }\end{array}$} \\
\hline & & & & NES & $P$-value & NES & $P$-value & $\mathrm{Z}$ & $P$-value & NES & $P$-value & $\mathrm{Z}$ & $P$-value \\
\hline 6: 33043976-33054001 & 56 & $H L A-D P B I$ & Body & -2.08 & $3.45 \mathrm{E}-05$ & -1.98 & $8.06 \mathrm{E}-05$ & -5.69 & $1.25 \mathrm{E}-08$ & -1.05 & $3.51 \mathrm{E}-01$ & -5.43 & 5.46E-08 \\
\hline 6: 33048416-33048814 & 17 & $H L A-D B P 1$ & Island & -2.04 & $6.96 \mathrm{E}-05$ & -2.25 & $1.62 \mathrm{E}-04$ & -5.46 & $4.80 \mathrm{E}-08$ & -1.16 & $2.15 \mathrm{E}-01$ & -5.38 & 7.49E-08 \\
\hline $21: 35831697-35832365$ & 10 & KCNE1 & Island & -1.93 & $1.32 \mathrm{E}-04$ & -2.16 & $1.72 \mathrm{E}-04$ & -5.33 & 9.99E-08 & -1.27 & $1.58 \mathrm{E}-01$ & -5.34 & 8.93E-08 \\
\hline $21: 35827824-35884508$ & 23 & KCNE1 & Promoter & -1.93 & $9.45 \mathrm{E}-05$ & -2.00 & $3.42 \mathrm{E}-04$ & -5.28 & 1.27E-07 & -1.22 & $1.91 \mathrm{E}-01$ & -5.26 & 1.46E-07 \\
\hline 6: 32547019-32557404 & 34 & HLA-DRBI & Body & -1.23 & $2.00 \mathrm{E}-01$ & -2.48 & $1.67 \mathrm{E}-05$ & -3.67 & $2.43 \mathrm{E}-04$ & -2.58 & $2.62 \mathrm{E}-05$ & -5.24 & 1.58E-07 \\
\hline 7: 1885033-1885402 & 3 & MADIL1 & Island & -2.22 & $1.81 \mathrm{E}-05$ & -2.12 & $1.72 \mathrm{E}-04$ & -5.69 & $1.25 \mathrm{E}-08$ & -0.81 & $7.38 \mathrm{E}-01$ & -5.15 & 2.65E-07 \\
\hline 7: 27169572-27170638 & 10 & HOXA4 & Island & -2.21 & $1.73 \mathrm{E}-05$ & -1.98 & $3.26 \mathrm{E}-04$ & -5.60 & 2.15E-08 & -0.84 & $7.46 \mathrm{E}-01$ & -5.06 & 4.20E-07 \\
\hline 8: $125461772-125464547$ & 9 & TRMT12 & Promoter & -2.20 & $1.85 \mathrm{E}-05$ & -1.14 & $2.98 \mathrm{E}-01$ & -4.01 & $6.11 \mathrm{E}-05$ & -1.93 & $1.55 \mathrm{E}-03$ & -5.03 & 4.69E-07 \\
\hline 7: 27169740-27171528 & 24 & HOXA4 & Promoter & -2.31 & $1.78 \mathrm{E}-05$ & -1.97 & $3.41 \mathrm{E}-04$ & -5.59 & 2.31E-08 & -0.68 & $9.18 \mathrm{E}-01$ & -4.94 & 7.71E-07 \\
\hline $6: 25882327-25882560$ & 4 & SLC17A3 & Island & -1.96 & $9.39 \mathrm{E}-05$ & -1.50 & $4.91 \mathrm{E}-02$ & -4.29 & $1.81 \mathrm{E}-05$ & -1.63 & $3.04 \mathrm{E}-02$ & -4.80 & $1.60 \mathrm{E}-06$ \\
\hline 1: $156814881-156815792$ & 5 & NTRK1 & Island & -1.51 & $5.29 \mathrm{E}-02$ & -1.78 & $3.74 \mathrm{E}-03$ & -3.31 & $9.20 \mathrm{E}-04$ & -2.12 & $1.16 \mathrm{E}-04$ & -4.76 & 1.90E-06 \\
\hline 16: 1561036-1652552 & 121 & IFT140 & Body & -1.67 & $7.56 \mathrm{E}-04$ & -1.82 & $5.99 \mathrm{E}-05$ & -5.12 & 2.92E-07 & -0.83 & $8.80 \mathrm{E}-01$ & -4.57 & $4.96 \mathrm{E}-06$ \\
\hline 17: 8700574-8703341 & 12 & MFSD6L & Promoter & -2.33 & $1.84 \mathrm{E}-05$ & -1.82 & $3.56 \mathrm{E}-03$ & -5.17 & $2.36 \mathrm{E}-07$ & 1.03 & $4.10 \mathrm{E}-01$ & -4.52 & $6.33 \mathrm{E}-06$ \\
\hline 16: $1583809-1584641$ & 8 & IFII40 & Island & -2.46 & $1.81 \mathrm{E}-05$ & -1.79 & $2.75 \mathrm{E}-03$ & -5.22 & 1.78E-07 & 0.69 & $8.80 \mathrm{E}-01$ & -4.50 & $6.79 \mathrm{E}-06$ \\
\hline
\end{tabular}




\begin{tabular}{|c|c|c|c|c|c|c|c|c|c|c|c|c|c|}
\hline 5: 191792-192544 & 5 & LRRC14B & Island & -2.19 & $1.81 \mathrm{E}-05$ & -1.57 & $2.34 \mathrm{E}-02$ & -4.77 & 1.83E-06 & -0.93 & $5.50 \mathrm{E}-01$ & -4.47 & $7.96 \mathrm{E}-06$ \\
\hline 6: 168433191-1.68E+08 & 18 & KIF25 & Body & -2.13 & $5.45 \mathrm{E}-05$ & -1.79 & $4.11 \mathrm{E}-03$ & -4.94 & 7.59E-07 & -0.62 & $9.50 \mathrm{E}-01$ & -4.36 & $1.30 \mathrm{E}-05$ \\
\hline 10: 530713-531099 & 5 & DIP2C & Island & -2.22 & $3.54 \mathrm{E}-05$ & -1.98 & $5.05 \mathrm{E}-04$ & -5.40 & $6.63 E-08$ & 1.148 & $2.60 \mathrm{E}-01$ & -4.19 & $2.78 \mathrm{E}-05$ \\
\hline 1: 2986362-3349982 & 608 & PRDM16 & Body & -1.73 & $1.34 \mathrm{E}-05$ & -1.43 & $2.06 \mathrm{E}-04$ & -5.71 & 1.09E-08 & 1.168 & $8.00 \mathrm{E}-02$ & -4.15 & $3.25 \mathrm{E}-05$ \\
\hline 17: 8702342-8702824 & 7 & MFSD6L & Island & -2.19 & $1.84 \mathrm{E}-05$ & -1.82 & $2.42 \mathrm{E}-03$ & -5.24 & 1.59E-07 & 0.684 & $8.70 \mathrm{E}-01$ & -4.13 & $3.57 \mathrm{E}-05$ \\
\hline 12: $9217328-9217715$ & 6 & LINC00612 & Island & -2.27 & $1.84 \mathrm{E}-05$ & -2.36 & $1.75 \mathrm{E}-04$ & -5.69 & 1.30E-08 & 1.465 & $6.00 \mathrm{E}-02$ & -4.09 & $4.31 \mathrm{E}-05$ \\
\hline 12: 9217079-9217769 & 9 & LOC144571 & Promoter & -2.27 & $1.85 \mathrm{E}-05$ & -2.16 & $1.81 \mathrm{E}-04$ & -5.68 & $1.34 \mathrm{E}-08$ & 1.530 & $4.00 \mathrm{E}-02 *$ & -4.00 & $6.46 \mathrm{E}-05$ \\
\hline 11: 70672834-70673055 & 6 & SHANK2 & Island & -2.56 & $1.83 \mathrm{E}-05$ & -2.32 & $1.73 \mathrm{E}-04$ & -5.69 & 1.28E-08 & 1.752 & $6.00 \mathrm{E}-03 *$ & -3.67 & $2.45 \mathrm{E}-04$ \\
\hline 17: 76037074-76037323 & 3 & TNRC63 & Island & -2.03 & $9.29 \mathrm{E}-05$ & -2.05 & $1.78 \mathrm{E}-04$ & -5.39 & 7.07E-08 & 1.693 & $1.10 \mathrm{E}-02 *$ & -3.49 & $4.82 \mathrm{E}-04$ \\
\hline
\end{tabular}

Chr: chromosome, NES: normalized effect score. All positions and regions were in reference to GRCh37/hg19. Significance is indicated in bold. The asterisks indicate nominal significance in PRISMO. The $p$-values for MRS, Army STARRS and the meta-analyses are Bonferroni-corrected for $\sim 26 \mathrm{~K}$ DMRs. The discovery stage meta-analysis includes MRS and Army STARRS while the last column also includes PRISMO. The table is organized based on significance of the DMRs in the stage 2 meta-analysis.

\begin{tabular}{|l|l|l|l|}
\hline \multicolumn{4}{|l|}{ Table 4. Correlations between methylation levels of DMPs and gene expression data from MRS } \\
\hline CpG & Gene & Correlation $(r)$ & $p$-value \\
\hline cg20756026 & HEXDC & -0.27 & $\mathbf{1 . 4 8 E - 0 5}$ \\
\hline $\operatorname{cg} 12169700$ & MAD1L1 & 0.15 & $\mathbf{0 . 0 1 4}$ \\
\hline $\operatorname{cg} 05656210$ & SPRY4* & 0.09 & 0.16 \\
\hline $\begin{array}{l}\text { Nominal significance is indicated in bold. } \\
* \text { Closest gene }\end{array}$
\end{tabular}




\section{EWAS DMP}
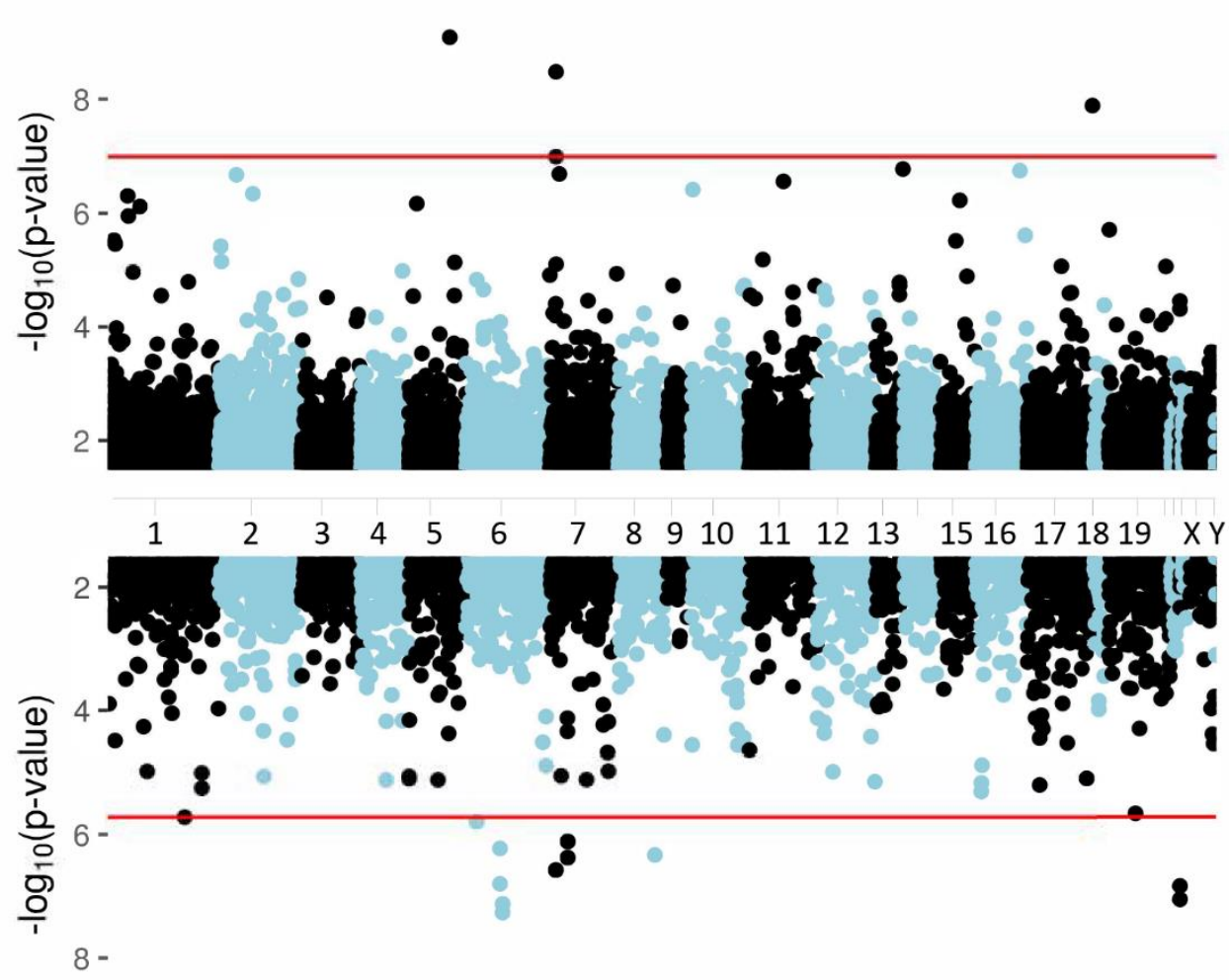

\section{EWAS DMR}

Figure 1. Manhattan plot showing a meta-analysis across 3 epigenome-wide association studies (MRS, Army STARRS, PRISMO). The upper part shows the 3 significant differentially methylated positions (DMPs) while the lower part shows the 12 significant differentially methylated regions (DMRs). Red lines indicate significance thresholds after Bonferroni corrections for 485,000 (top) and 26,000 (bottom) comparisons, respectively. 